\title{
Study of Biochemical Profile of HbA1c, Vitamin D and AMH in Infertile Women of a Population of South India
}

\author{
${ }^{1}$ Asssociate Professor, Department of Biochemistry, SSMC, Tumkur, India \\ ${ }^{2}$ Assistant Professor/Statistician, Department of Community Medicine, SSMC, Tumkur, India \\ ${ }^{3}$ Associate Professor, Department of Physiology, SSMC, Tumkur, India \\ ${ }^{4}$ Assistant Professor, Department of Physiology, SSMC, Tumkur, India
}

Sathisha $\mathrm{TG}^{1}$, Bhargavi S K ${ }^{1 *}$, Riyaz Ahmad Kalaburgi ${ }^{2}$, Shashiraj $\mathrm{H} \mathrm{K}^{3}$, Kavitha BS ${ }^{4}$, Arifuddin $\mathrm{M} \mathrm{K}^{4}$

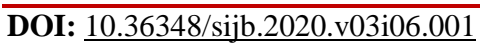

| Received: 11.05.2020 | Accepted: 18.05.2020 | Published: 12.06.2020

*Corresponding author: Dr. Bhargavi S K

\section{Abstract}

Infertility has grown into an emergent issue in recent times. It is defined as the inability of the couple to conceive despite two years of co habitation and unprotected exposure to pregnancy. As there is a dearth of studies concerning infertility among rural population this study was taken up in Sri Siddhartha Medical College, a constituent college of SSAHE, a Deemed to be university which caters to rural population of Tumkur district of Karnataka with the aim of assessing the role of HbA1c, Vitamin D and AMH in infertile women of the above mentioned population. Our case control study included age matched 40 infertile women and 40 apparently healthy women. Quantification of $\mathrm{HbA}_{1 \mathrm{c}}$ was by fluorescence immunoassay on the Fine Care analyzer. AMH was assayed on cobas e immunoassay analyzer adopting chemiluminescene method. Vitamin D estimated by fully automated chemiluminescene analyzer Siemens Advia Centaur for 25-OH Vitamin D. Our study revealed statistically significant $(\mathrm{P}-<0.001)$ increase of Blood HbA1c and serum AMH whereas significant $(\mathrm{P}-<0.001)$ decrease in serum Vitamin D levels. The present study shows that there is high prevalence of vitamin D deficiency, significantly elevated HbA1c levels which is marker of long term glycemic control and increased AMH levels which indicated anovulation in infertile women. So it is suggested that early estimation of these biochemical parameters and their correction would possibly lead to increased chances of conception in these infertile women of rural population.

Keywords: HbA1c, Vitamin D, AMH, glycemic control, anovulation, Infertile Women.

Copyright @ 2020: This is an open-access article distributed under the terms of the Creative Commons Attribution license which permits unrestricted use, distribution, and reproduction in any medium for non-commercial use (NonCommercial, or CC-BY-NC) provided the original author and source are credited.

\section{Study of Biochemical Profile of HbA1c, Vitamin D and AMH in Infertile women of a Population of South India}

Infertility has grown into an emergent issue in recent times. It is defined as the inability of the couple to conceive despite two years of co habitation and unprotected exposure to pregnancy. Clinically infertility is defined as failure to achieve pregnancy after one year or more of regular unprotected sexual intercourse [1].

Infertility is classified as primary or secondary where primary infertility is infertility where there has been no conception at all and secondary infertility is failure of conception following a previous pregnancy. The causes for infertility are varied from genetic, environmental and other infectious diseases. There is also increased incidence of infertility in couples who postpone parenthood, couples who are exposed to stress and other life style issues including obesity [2].
It is estimated that the global incidence of infertility varies from $08-12 \%$ [3] and also the estimates suggest that the burden of infertility is almost three times higher in developing countries in comparison to developed part of world. According to Indian society of Assisted Reproduction, infertility currently affects about $10-14 \%$ of Indian population with higher rates in urban areas where in one out of six couples are impacted[4].

The estimate also suggests that 27.5 million couples actively trying to conceive suffer from infertility in India. Infertility exposes the couple to intense community pressure to produce a biological child which leads to public health issues with serious social consequences. Recent data suggests that the incidence of infertility is increasing in rural population of India. The social consequences of not having children leads to a sense of loss which makes the couple view infertility as a tragedy. This leads to social 
implications which affect women to a greater degree when compared to men [5].

Definitely an amplified risk of glucose
metabolism disorders exists in women with
reproductive disorders. Such women have the
prevalence of pre-DM is $35 \%$ and the prevalence of
DM can be as high as $10 \%$ [6]. HbA1C quantifies the
amount of glycated hemoglobin in the blood, which
evaluates long-term glycemic exposure.

Vitamin D is named as Sunshine Hormone due to its synthesis in the presence of UV -rays of the Sun. It's a fat soluble vitamin derived from cholesterol. Follicular development, differentiation and luteinization are significant functions of Vitamin D. Different organs express Vitamin D Receptor (VDR) through which Vitamin D enacts a major role. Vitamin D influences fertility and pregnancy. As suggested by studies, Vitamin D functions in infertility by VDR receptor expression on both male and female reproductive systems [7]. Paucity of Vitamin D is paired with infertility.

AMH (Anti Mullerian Hormone), a dimeric glycoprotein, belongs to the transforming growth factor- $\beta$ family and is secreted almost exclusively by the granulosa cells of the early antral follicles. AMH plays a role in folliculogenesis by inhibiting primordial follicular recruitment and FSH-dependent growth and selection of antral follicles. It is an established indicator of ovarian reserve and predictor of ovarian response in Assisted Reproductive Technologies (ART) [8]. Serum AMH levels are high in women with infertility due to an increase in the number of small antral follicles and an intrinsic defect of the granulosa cells. Ethnicity has been associated with altered agespecific levels of AMH, with Asian women having a lower $\mathrm{AMH}$ at a given age compared to their Caucasian counterparts [9].

It is implied that there is derangement of biochemical profile of Vitamin D, HbA1c which quantifies the amount of glycated hemoglobin in the blood and is a marker of glycemic control. AMH which is an established marker of ovarian reserve tends to be high in women with infertility.

As there is a dearth of studies concerning infertility among rural population the present study has been taken up in Sri Siddhartha Medical College, a constituent college of SSAHE, a Deemed to be university which caters to rural population of Tumkur district of Karnataka with the aim to assess the role of HbA1c, Vitamin D and AMH in infertile women of the above mentioned population.

\section{MATERIALS AND METHODS}

Our case control study included age matched 40 infertile women and 40 apparently healthy women. Control group consisted of arbitrarily chosen women. Study population included was from rural area of Tumkur, Karnataka attending to SSMC Hospital. The time duration of study was from 2018-2019. The institutional ethics committee approved the study on human experimentation in accordance with the latest version of Helsinki Declaration. Every subject gave written informed consent.

\section{Inclusion Criteria}

Newly diagnosed female infertile patients and controls in the age group 20-40 years.

\section{Exclusion Criteria}

Female patients or controls suffering from diabetes, hypertension and any other endocrine disorders, patients who had already received or were under treatment for infertility were excluded from study. Clinical investigations and questionnaires formed the basis of enquiry.

\section{Collection of blood}

With strict aseptic precautions, $5 \mathrm{ml}$ of fasting venous blood was drawn from median cubital/basilic vein on 2-5 day of menstrual cycle i.e. early follicular phase. Later $3 \mathrm{ml}$ of blood was transferred into BD red capped plain vacutainers and remaining $2 \mathrm{ml}$ into BD EDTA vacutainers. Plain Vacutainers were allowed to stand for $10 \mathrm{~min}$ at room temperature for clotting. At 3000rpm they were centrifuged for 10 minutes using Remi8RC centrifuge. Separation of serum was done. All the parameters were estimated on the same day.

Quantification of $\mathrm{HbA}_{1 \mathrm{c}}$ was done by fluorescence immunoassay on the Fine Care analyzer. AMH was assayed on cobas e immunoassay analyzers (Roche Diagnostics GmbH, Germany) adopting chemiluminescene method. Vitamin D was estimated by fully automated chemiluminescene analyzer Siemens Advia Centaur for 25-OH Vitamin D.

\section{STATISTICAL ANALYSIS}

The data was entered in excel spread sheet. Data cleaning and validation was done and analyzed using Statistical Package for Social Sciences (SPSSVersion 20) (IBM Corp., Armonk, NY, USA). All variables were tested for normality with the help of Kolmogorov-Smirnov test. Data was presented as Mean and Standard deviation for continuous variables. Variables were compared using an independent sample $\mathrm{t}$-test for normally distributed data. P-value $<0.05$ was considered statistically significant. 


\section{RESULTS}

Table-1: Comparison of HbA1c, Vitamin D and AMH between infertile and healthy women

\begin{tabular}{|c|c|c|c|c|c|c|}
\hline Parameter & Group & $\mathbf{N}$ & Mean & Std. Deviation & t- value & P-value \\
\hline \multirow{2}{*}{$\begin{array}{l}\text { HbA1c } \\
(\%)\end{array}$} & Control & 40 & 5.13 & 0.59 & \multirow{2}{*}{-9.429} & \multirow{2}{*}{$<0.001$ (Significant) } \\
\hline & Cases & 40 & 7.47 & 1.46 & & \\
\hline \multirow{2}{*}{$\begin{array}{l}\text { Vitamin D } \\
(\mathrm{ng} / \mathrm{ml})\end{array}$} & Control & 40 & 62.00 & 20.14 & \multirow{2}{*}{14.869} & \multirow{2}{*}{$<0.001$ (Significant) } \\
\hline & Cases & 40 & 14.07 & 3.13 & & \\
\hline \multirow{2}{*}{$\begin{array}{l}\text { AMH } \\
(\mathrm{ng} / \mathrm{ml})\end{array}$} & Control & 40 & 4.03 & 0.74 & \multirow{2}{*}{-11.756} & \multirow{2}{*}{$<0.001$ (Significant) } \\
\hline & Cases & 40 & 7.12 & 1.49 & & \\
\hline
\end{tabular}

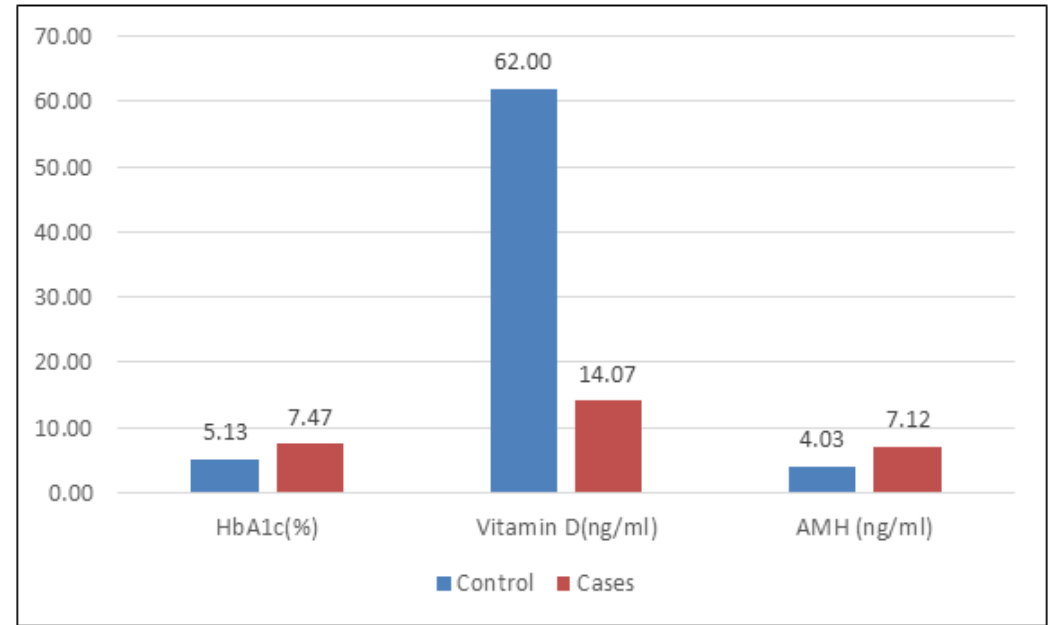

Graph-1: Comparison of HbA1c, Vitamin D and AMH between control and cases

Our study revealed statistically significant increase of Blood $\mathrm{HbA1c}$ and serum AMH whereas significant decrease in serum Vitamin D levels.

\section{DISCUSSION}

A significant portion of infertility may at least partially be explained by exposure to environmental factors, endocrine disruptions, and hormonal imbalances.

Polycystic Ovarian Syndrome (PCOS) is one of the most widespread endocrine system disorders that distress women of reproductive age with a prevalence of $8-13 \%$ [10]. Women with PCOS are double prone to be hospitalized against normal .Hence precise and premature diagnosis of PCOS is need of the hour which curtails potential health hazards and financial expenditure. The ubiquitous presenting element of PCOS is Infertility. Approximately $75 \%$ of such women are anovulating hence PCOS is the most frequent cause of anovulatory infertility [11].

Current study results demonstrate HbA1c was elevated in a higher number of patients in comparison to controls [12].Natural non -enzymatic glycation of hemoglobin occurs on exposure to plasma glucose which yields HbA1c. Concentration of the glucose in the blood directly influences the rate of formation of HbA1c which reflects the cumulative values of glucose during the last 8-12 weeks. An unstable Schiff base is formed by condensation of glucose with the N-end of each beta chain of $\mathrm{Hb}$. A stable ketomine termed as HbA1c is produced by an amdori reshuffling,an irreversible process. Glucose concentration and lifespan of $\mathrm{RBC}$ in the plasma are the regulators of $\mathrm{HbA} 1 \mathrm{c}$.

$\mathrm{HbA} 1 \mathrm{C}$ is a fast and convenient method for diagnosing pre-DM in the general adult population. According to American Diabetes Association (ADA) $2013 \mathrm{HbA} 1 \mathrm{C}$ is an acceptable method for the diagnosis of pre-DM in the general population [13]. When women visit Infertility center, it is a chance to discover the preDM in disguise. Perhaps such women are undiagnosed as they have no symptoms at all. Individuals having $\mathrm{HbA} 1 \mathrm{C}$ of $6 \%$ to $6.5 \%$ pose a 5 -year risk of $25 \%$ to $50 \%$ of developing DM which can be critically intervened by early identification of patients with preDM[14].

The present study revealed that a greater part of patients had a deficit of Vitamin D. Such results are in agreement with the results of previous studies demonstrating vitamin $\mathrm{D}$ dearth in reproductive age women $[15,16]$. India is a tropical country. Though there is no scarcity of sunlight, Vitamin D deficiency is prevalent in women as mostly they stay indoors [17]. Vitamin D deficiency is related to many diseases. It predisposes to diseases or accentuates the disease status. Vitamin D status of patients needs notice [18]. One of the ailments of Vitamin D deficiency is 
Infertility. Vitamin D deficit is highly probable in infertile women [19]. The scarcity of Vitamin D needs to be recognized and supplemented for the same.

Our study exposed a higher level of serum $\mathrm{AMH}$ in cases when compared to controls. Anovulatory infertility is mostly caused by polycystic ovarian syndrome (PCOS). It is multifactorial as well as polygenic and perhaps the most frequent endocrine disorder among the reproductive age women[20]. Considerable aberrations of peri-follicular vascularity, granulosa cell function and abnormal circulating hormones are related to Polycystic ovary syndrome (PCOS). Developmental competence as well as deprived oocyte quality is concerned with high granulosa cell apoptosis, changed intrafollicular environment, heterogeneity of steroidogenesis amid every follicles and oxidative stress leading to mitochondrial damage and dysfunction as well as epigenetic modification[21].

Women with ovulatory PCOS generate 20 folds more AMH whereas those with anovulatory PCOS produce around 75 times higher per granulosa cell in comparison to healthy controls[22]. Significantly elevated serum AMH levels in PCOS women was apparent in contrast to controls in Caucasian women [23], as well other ethnic groups[24] which was corroborated by diverse authors. Our study findings are in accordance with above authors.

\section{CONCLUSION}

The present study shows that there is a high prevalence of Vitamin D deficiency, significantly elevated HbA1c levels which is a marker of long term glycemic control and increased AMH levels which indicated anovulation in infertile women. So it is suggested that early estimation of these biochemical parameters and their correction would possibly lead to increased chances of conception in these infertile women of rural population.

\section{REFERENCES}

1. Zegers-Hochschild, F., Adamson, G. D., de Mouzon, J., Ishihara, O., Mansour, R., Nygren, K., ... \& Van der Poel, S. (2009). The international committee for monitoring assisted reproductive technology (ICMART) and the world health organization (WHO) revised glossary on ART terminology, 2009. Human reproduction, 24(11), 2683-2687.

2. Schmidt, L., Sobotka, T., Bentzen, J. G., Nyboe Andersen, A., \& ESHRE Reproduction and Society Task Force. (2012). Demographic and medical consequences of the postponement of parenthood. Human reproduction update, 18(1), 29-43.

3. World Health Organization (WHO). (1999). Infertility: A Tabulation of Available Data on
Prevalence of Primary and Secondary Infertility. WHO/MCH/91.9.Geneva: World Health Organization.

4. Ganguly, S., Unisa, S. (2010). Trends of infertility and childlessness in India: findings from NFHS data. Facts Views Vis Obgyn, 2(2), 131-138.

5. Bharadwaj, A. (2002). Culture, infertility and gender-vignettes from South Asia and North Africa. Sex Health Exch, 2, 14-15.

6. Ehrmann, D. A., Barnes, R. B., Rosenfield, R. L., Cavaghan, M. K., \& Imperial, J. (1999). Prevalence of impaired glucose tolerance and diabetes in women with polycystic ovary syndrome. Diabetes care, 22(1), 141-146.

7. Ranjana, H. (2017). Role of vitamin D in infertility. J Public Health Policy Plann, $1,1$.

8. Tal, R., \& Seifer, D. B. (2017). Ovarian reserve testing: a user's guide. American journal of obstetrics and gynecology, 217(2), 129-140.

9. Iglesias, C., Banker, M., Mahajan, N., Herrero, L., Meseguer, M., \& Garcia-Velasco, J. A. (2014). Ethnicity as a determinant of ovarian reserve: differences in ovarian aging between Spanish and Indian women. Fertility and sterility, 102(1), 244249.

10. Azziz, R., Woods, K. S., Reyna, R., Key, T. J., Knochenhauer, E. S., \& Yildiz, B. O. (2004). The prevalence and features of the polycystic ovary syndrome in an unselected population. The Journal of Clinical Endocrinology \& Metabolism, 89(6), 2745-2749.

11. Homburg, R. (2004). Management of infertility and prevention of ovarian hyperstimulation in women with polycystic ovary syndrome. Best Practice \& Research Clinical Obstetrics \& Gynaecology, 18(5), 773-788.

12. de Medeiros, S. F., Yamamoto, M. M. W., Bueno, H. B., Belizario, D., \& Barbosa, J. S. (2014). Prevalence of elevated glycated hemoglobin concentrations in the polycystic ovary syndrome: anthropometrical and metabolic relationship in Amazonian women. Journal of clinical medicine research, 6(4), 278.

13. American Diabetes Asociation. (2009). Standards of medical care in diabetes. Diabetes Care. Actualización Médica Periódica, 32(s1).

14. Zhang, X., Gregg, E. W., Williamson, D. F., Barker, L. E., Thomas, W., Bullard, K. M., ... \& Albright, A. L. (2010). A1C level and future risk of diabetes: a systematic review. Diabetes care, 33(7), 1665-1673.

15. Tabrizi, R., Moosazadeh, M., Akbari, M., Dabbaghmanesh, M. H., Mohamadkhani, M., Asemi, Z., ... \& Lankarani, K. B. (2018). High prevalence of vitamin d deficiency among iranian population: A systematic review and metaanalysis. Iranian journal of medical sciences, 43(2), 125.

16. Pagliardini, L., Vigano, P., Molgora, M., Persico, P., Salonia, A., Vailati, S. H., ... \& Candiani, M. 
(2015). High prevalence of vitamin D deficiency in infertile women referring for assisted reproduction. Nutrients, 7(12), 9972-9984.

17. Hassannia, T., GhaznaviRad, E., Vakili, R., Taheri, S., \& Rezaee, S. A. (2015). High prevalence of vitamin D deficiency and associated risk factors among employed women in a sunny industrial city. Int. J. Vitam. Nutr. Res, 85(3-4), 119-128.

18. Anderson, J. L., May, H. T., Horne, B. D., Bair, T. L., Hall, N. L., Carlquist, J. F., ... \& Group, I. H. C. I. S. (2010). Relation of vitamin D deficiency to cardiovascular risk factors, disease status, and incident events in a general healthcare population. The American journal of cardiology, 106(7), 963-968.

19. Lerchbaum, E., Rabe, T. (2014). Vitamin D and female fertility. Curr Opin Obstet Gynecol, 26, 145-150.

20. Kousta, E., White D.M., Franks, S. (1997). Modern use of clomiphene citrate in induction of ovulation. Hum Reprod Update, 3, 359-365.

21. Eini, F., Novin, M. G., Joharchi, K., Hosseini, A., Nazarian, H., Piryaei, A., \& Bidadkosh, A. (2017).
Intracytoplasmic oxidative stress reverses epigenetic modifications in polycystic ovary syndrome. Reproduction, Fertility and Development, 29(12), 2313-2323.

22. Pellatt, L., Hanna, L., Brincat, M., Galea, R., Brain, H., Whitehead, S., \& Mason, H. (2007). Granulosa cell production of anti-Mullerian hormone is increased in polycystic ovaries. The Journal of Clinical Endocrinology \& Metabolism, 92(1), 240245.

23. Sahmay, S., Atakul, N., Aydogan, B., Aydın, Y., Imamoglu, M., \& Seyisoglu, H. (2013). Elevated serum levels of anti Müllerian hormone can be introduced as a new diagnostic marker for polycystic ovary syndrome. Acta obstetricia et gynecologica Scandinavica, 92(12), 1369-1374.

24. Chao, K. C., Ho, C. H., Shyong, W. Y., Huang, C. Y., Tsai, S. C., Cheng, H. Y., ... \& Li, H. Y. (2012). Anti-Mullerian hormone serum level as a predictive marker of ovarian function in Taiwanese women. Journal of the Chinese Medical Association, 75(2), 70-74. 